\title{
Portraits of participation: Exploring the relationship between social motivators and facets of participation in a Twitter-based community
}

\author{
Sarah Gilbert \\ iSchool \\ University of British Columbia \\ s.gilbert@ubc.ca
}

\begin{abstract}
Twitter is a platform where people can coalesce around a common interest, signaled by a hashtag, and form a community of practice. As with all online initiatives, questions remain about what motivates people to follow and contribute to communities, and why they participate in varying degrees. This paper explores social motivations for participation in the Twitter-based community of practice, \#hcsmca (Healthcare Social Media Canada), formed in 2010 to discuss issues in healthcare within a Canadian context. Analysis of 24 semi-structured interviews identified three important social motivations: tapping into a social network of people with a common interest, developing personal and professional relationships, and the community ethos. Portraits of participation based on three facets of participation, length of time as a community member, depth of engagement in the community, and frequency of participation, were developed to describe community members' motivations at varying levels of participation.
\end{abstract}

\section{Introduction}

Communities of practice are complex social structures that support learning and development of common practices and identity [32]. As online communities of practice have become more accepted, the media supporting them has shifted. The most recent of these shifts is to Twitter. One popular Twitter-based community structure is the tweet chat, where communities are formed around a shared interest that is signaled through a hashtag. Chat times are set in advance and through the conversational practices of tweeting, replying, and retweeting, the social routines of offline communities of practice transpire. Yet, as with all distributed, virtual communities, questions remain about what motivates individuals to follow, contribute and maintain such communities. As social interaction is a key element of online community success [21], are social motivators important to community members? Is there a relationship between the ways in which community members participate and social motivations? To explore the relationship between social motivations and participation, 24 interviews were conducted with members of the Health Care Social Media Canada (\#hcsmca) community, which, from September 2010 until March 2016, met weekly on Twitter to discuss the role of social media in Canadian healthcare via tweet chats.

This paper is part of a series of studies examining social interaction in social media communities, including a previous paper on \#hcsmca that provides an overview of social network interactions among community members [12], and an exploration of learning processes in \#hcsmca [10]. This paper focuses on social interaction in \#hcsmca, addressing the following two research questions:

- RQ1: What aspects of social interaction are important to participants of \#hcsmca?

- RQ2: How do different elements of participation, such as length of time as a community member, depth of engagement, and frequency of participation, relate to social motivations for participants of \#hcsmca?

Results of this research describe relationships between various aspects and degrees of participation and social motivators, which helps inform theoretical perspectives on community development and technology use, and has practical implications for those supporting and/or participating in Twitter-based communities, and online communities of practice.

\section{Theoretical background}

This work is informed by three areas of research: social network perspectives that provide insight into interaction effects; research on participation in online communities that provides perspectives on complex and overlapping features of online interaction; and motivation theories that provide insight into why people choose to participate in online initiatives. 


\subsection{Social network perspective}

The social network perspective provides both theories and an analytical framework for exploring and explaining dynamic interactions between people. From this perspective, social networks are described through two basic elements: actors in a network and the relations that connect them. Social effects build from these elements to show whole networks of resource access and flow, and the basis of self-sustaining networks that no longer depend on single actor input to remain active. Among the key features often discussed about communities and networks is the idea of social capital, i.e., the investment present in a community that can be tapped by individuals as participants of that network. Social capital is commonly characterized as either bridging or bonding capital. Bonding capital looks inward to the ties maintained within the network; it is associated with solidarity, aid, and reciprocity. Bridging capital is looks outward for ties that can bring resources into a network; it contributes to asset and information diffusion [29].

The concepts of bridging and bonding are tightly tied to the concept of tie strength. Strength is defined as a combination of the amount of time, intensity, intimacy, and reciprocal services shared between two actors [11]. Bonding capital is maintained through strong tie relationships while bridging capital arises from weak tie relationships [11]. In addition to weak and strong ties, latent ties occur between actors who could, but have yet to activate formal connections [13]. Community networks typically consist of actors with both weak and strong ties and therefore may benefit from varying degrees of both bridging and bonding capital; the former keep the community strong, the latter keep the community informed and aware of external activity.

Social network ties can be built and strengthened through media use. In online and offline environments [13] found that tie strength is associated with media use: strongly tied pairs use more media to communicate than weakly tied pairs. Weakly tied pairs use public media to communicate while strongly tied pairs use public media in addition to private media. For example, in an online class, weakly tied pairs only used the public chat room to communicate while strongly tied pairs communicated via the public chat room and privately online and/or on the telephone. This phenomenon, and the ability of the public forum to act as a place for ties to be build from a latent tie structure, is referred to as media multiplexity [13].

Social capital may be built through social media use. For example, [8] found a positive relationship between Facebook use and the maintenance and development of bridging and bonding social capital. In
Twitter, [17] found that bridging capital was associated with a high level of followees who provide access to diverse information while bonding capital was associated with having a high number of followers who provide a feeling of support. While both types of social capital were supported, participants reported higher levels of bridging than bonding social capital.

\subsection{Participation in online communities}

Tie strength and the advantages of bridging and bonding social capital provide insight into relationships between members within a community, but stop short of describing the variety of engagement within communities. Our understanding of participation in online communities is becoming more multifaceted, particularly as we mix community associations across geography, media, and purpose. In particular, a number of studies have highlighted the kind of emerging roles seen in online communities [15].

One of the most commonly used models of varying participation in online initiatives is the Reader to Leader framework [28]. Readers do not contribute content, but may regularly follow websites for years. Contributors participate by adding small contributions; in a tweet chat, these take the form of favouriting tweets, retweeting, or sharing information using a hashtag. While contributions are minimal, they increase social presence within a community. Collaborators engage more with others through their communication behaviours; in tweet chats they respond to others' posts, and use@mentions to identify others. Through collaboration, community members develop mutual understanding, trust, and willingness to reciprocate. Leaders are most active and are responsible for the maintenance of the community. In a tweet chat Leaders organize, sustain, and plan the community and are among the community's most respected members. Typically, these levels of participation develop in a sequence, although for some participation may terminate, plateau, or decrease [28].

Another framework that addresses variations in participation is light and heavyweight models of peer production [14]. In this model, weight is used to describe contributors' commitment to and engagement with the project and other contributors. Lightweight models are typically associated with crowdsourcing initiatives where the accumulation of input relies on minimal, easy, rule-based contributions from a large pool of independent participants; for example, classifying galaxy types for the citizen science project Galaxy Zoo. Those who contribute to lightweight models are often driven by personal interest, orientation to the project ethos, and minor recognition for contribution. Heavyweight models are typically associated with communities. Contributions to 
communities are more substantial, collaborative, and made with attention to others' input. Heavyweight models support social interactions in which shared norms are developed. Online communities such as \#hcsmca aim for this kind of internal attention and commitment and thus is an example of a heavyweight model. Those who contribute to heavyweight models are also often driven by personal interest and orientation to the project ethos, but also by recognition for the quality of their efforts and the opportunity to build their reputation. In the design of such initiatives, those that might be classed as following a lightweight model focus on the individual and their contribution; by contrast, an initiative that focuses on following a heavyweight model models puts focus on facilitating interpersonal interaction and group maintenance.

While light and heavyweight models may apply to entire project structures, it can also describe participation within projects [14]. For example, in Wikipedia lightweight participation is demonstrated by those who offer small edits, while heavyweight participation is demonstrated by crafting articles and engaging with others in talk page discussions. Likewise, in \#hcsmca, community members participate at varying weights; the various facets of participation weight in \#hcsmca are outlined in the Methodology section below. For the remainder of the paper the term "weight" is used to describe the overall degree of participation intensity within communities.

Despite the nuances of roles, practices, and levels of participation in online communities, a review by [24] shows that empirical research investigating participation still commonly measures participation by quantity, despite evidence that it is often passive [24] and that lurkers account for a significant proportion of community members [21]. Participation is often examined in a single dimension, such as the degree to which participants contribute [e.g., 9, 4, 22], tenure in the community [e.g., 26, 34] or how frequently they contribute [e.g, 25]. While these studies have found that participation at different levels and roles arise from a variety of reasons, few studies explore multiple dimensions of participation and their relationship to motivation [e.g., 26]. Examining motivations at these different levels of participation can fill this gap in the literature and add significantly to our understanding of contemporary community practice.

\subsection{Motivations for participating in an online community}

One of the most commonly used frameworks for exploring motivations for participating in online communities is Self-determination theory [30]. Selfdetermination theory divides motivations into two categories: intrinsic and extrinsic. Intrinsic motivations are inherently pleasing (such as having fun and learning) while extrinsic motivations lead to an external reward (such as enhanced reputation and financial gain) [30]. To motivate online contribution [2] emphasizes the importance of appealing to intrinsic motivators, although acknowledges that all incentives will not work for the same people at all times. Indeed, studies employing self-determination theory have found participation in a variety of initiatives is driven by a combination of intrinsic and extrinsic motivators. For example, [3] found that participants in a crowdsourcing project were motivated by a combination of intrinsic and extrinsic motivators including career, recognition, contribution to a collaborative effort, and self expression. In Mechanical Turk, [19] found that extrinsic motivators were associated with time spent on the platform, but intrinsic motivations were perceived as more important.

Participation weight has been found to also affect motivation. For example, [4] found that in OpenStreetMap, serious mappers (i.e., heavyweight participants) were more oriented to community, learning, and career motivations while casual mappers (lightweight participants) were more oriented to the principles of freely available mapping data. In the citizen science project, Old Weather, [9], found that intrinsically motivated volunteers contributed in more depth than extrinsically motivated volunteers. As findings from these studies indicate a relationship between participation weight and motivation, the next step is to explore relationships between different aspects of participation and motivation.

\section{Methodology}

A case study approach was taken to achieve an indepth exploration of motivation, with \#hcsmca selected as a representative of a successful online community. \#hcsmca was founded by Colleen Young (@)colleen_young) in September 2010 and ran under her guidance until March 2016. The original intent was for a forum to discuss the intersection of social media and healthcare, but expanded to include discussions on an array of healthcare issues within a Canadian context. Discussions took place primarily on Twitter, but information regarding weekly chats was published on a blog run by Young, and regular offline meet-ups supplemented the tweet chats. The tweet chats were scheduled weekly and lasted an hour. The community met this way from inception until March 2016, when Young stepped down. Over the course of five and a half years 19,000 participants contributed 220,000 tweets to 252 tweet chats [33]. 
Each week guest moderators were invited to host chats devoted to a single topic. Hosts published a blog post on the topic and discussion questions were chosen in advance. The chats began with introductions, proceeded through the questions, and closed with a call for final thoughts. Hosts were guided through the process by Young. While weekly tweet chats are no longer taking place, the \#hcsmca hashtag is active and an offline offshoot of the community, EveningRounds, meets monthly.

\subsection{Data collection and analysis}

Data were collected through semi-structured interviews that focused on community members' interpretations of what motivated them to participate. The research design received approval from the University of British Columbia Behavioural Research Ethics Board. To introduce the study to the community I published a blog post and hosted a tweet chat. After hosting the chat I continued to participate in the community discussions at a low level; I introduced myself at the beginning of the chat, Tweeted several times during the chat, and responded to the call for final thoughts. While participation was solicited primarily through Twitter, Young sent a call for participation via email to known lapsed members. In all, 24 interviews were conducted, including an interview with Young. Interviews lasted an average of 45 minutes and were conducted via telephone (15), video conference (6), and in person (3); six participants were male and eighteen were female. Ten participants were healthcare communicators; five participants engaged in advocacy work, including providing a patient voice in the community; four participants were graduate students or academics; two were healthcare professionals; and three worked in other healthcarerelated fields.

During the interviews, participants were asked questions about their participation in the community, general motivations for participation, and about several motivators identified in previous studies on motivation: interest in the topic, learning, and relationship development. Participants were encouraged to describe the importance (or not) of group membership to them, and were asked to explain why and how the motivations discussed did (or did not) affect their participation in the group.

The data were analyzed following a grounded theory approach [7]. Themes related to social values held by community members were first identified and then reviewed to identify subthemes. Axial coding was carried out to identify patterns between the categories and three facets of participation. The first facet of participation, length describes for how long participants were members of the community. Five participants were Newbies (less than a year); eight participants were Regulars (1-3 years); and ten participants were Veterans, (4-5 years). The second facet, depth describes the level of engagement in the community and is based on the Reader to Leader framework [28]. Three participants were Readers who mostly lurked on chats or who only attended in person meet-ups; nine participants were Contributors who mostly favourited, re-tweeted, or posted single tweets using the hashtag; nine participants were collaborators who engaged in discussions with other community members and who have likely moderated chats; and three participants were Leaders who contributed to the development of the community. Finally, the third facet, frequency describes how often participants tuned into the chats. Five participants followed the chats Rarely (a few chats a year or less); eleven participants followed chats Sporadically (a few times per month or when the topic was relevant); and eight participants tuned in Habitually (nearly every week).

\section{Results}

The results are presented in two subsections. The first describes social drivers of participation in \#hcsmca and the second describes relationships between facets of participation and social motivation. Quoted participants were asked how they would like to be attributed and chose either their Twitter handle, first name, or a pseudonym [6].

\subsection{Social drivers of participation in \#hesmca}

In response to the first research question, "What aspects of social interactions are important to \#hcsmca community members?" three categories emerged from the data: ability to tap into the social network, relationship development, and the community ethos.

4.1.1. Tapping into the social network. Accessing the social network was a nearly ubiquitous motivator for \#hcsmca community members and was cited by 23 of the 24 participants. Participants identified six benefits related to accessing the network: connecting with people who have similar interests; having the opportunity to have their voices heard; gaining an overview of the field; access to diverse stakeholders within healthcare; connecting with influential people in healthcare; and getting feedback on their ideas.

Connecting with people who share similar interests was important to participants because it provided access to a community of practice and the opportunity to learn (for more on learning in \#hcsmca, see [10]). 
Kelly describes why she first began following the hashtag: "I used it a lot to find people who had either shared interest or who had a perspective that I was wanting to know more about." While communities of practice are commonly associated with professional practices, participants often described discussing their common interest as both personally and professionally relevant, and, when asked, many participants could not distinguish between the two. This is perhaps because people who voluntarily participate in communities of practice are passionate practitioners who care deeply about their profession.

Connecting to the network as a means of being heard was also an important motivator. For some, being heard was a way in which to promote their advocacy work. As@anetto describes: “Once I started getting more involved with healthcare and social media I started advocating for the voice of the patient to be involved." Others hoped their participation would help them become known in healthcare and could be a platform to demonstrate their interests and expertise.

On the receiver side, listening was a way to orient themselves in the healthcare field. @AdelineCohenB describes reading discussions as meaningful because: 'I'm relatively new to health care, so to me that's a way to understand the dialogues that are currently happening in the field." Orientation also provides participants with insight into roles within the field, which may be acted upon. As Eve describes:

"Lurking allows me to read who's writing what, when are they writing, what their positions are, their opinions, etc. and then if I want to engage, I can do so in a much more informed manner. Possibly even pursue something more, like a direct message to create a new connection, or even an offline meeting."

Learning is thus oriented to the subject matter (healthcare), and the wider community of practice around healthcare in Canada [10].

Social network analyses conducted by [12] showed that active \#hcsmca community members come from a variety of roles within healthcare. This diversity is an important aspect of participation for community members. Fostering professional diversity within the community was a specific goal of Young, who initially recruited members from diverse areas in healthcare as a way of "busting silos" within the field and to encourage intellectual cross pollination between professions. While diversity in role was most commonly cited by \#hcsmca community members, geographic diversity - a benefit of distributed communities of practice - also mattered. For @drpauldempsey from @quintepediatric, both aspects of diversity are important:

"it's one of the few ways of gathering a broad membership both in terms of interests, you know of patients, nurses, administrators, physiotherapists, doctors, etc., as well as geographically broad. There's great value in the geographic diversity of the community as well as the composition of the community."

While many \#hcsmca members reported diversity of the membership as providing access to diverse information and ideas, prior research suggests the caveat that Twitter users are unlikely to be exposed to cross-ideological content [16]. Indeed, some community members did express concern that topics were discussed within an echo-chamber. Such an issue reflects the usual dilemma of balancing access to new views and information (weak ties) with the benefits of critical mass for discussion (strong ties). As initial moderator, Young set up the conditions for what appear to be a reasonable balance between diversity and similarity. Yet, given the makeup of the group, it is likely that while roles of community members are diverse, their values are homogeneous.

Community members also valued the opportunity to interact with prestigious professionals in the field. @ carrield99 described \#hcsmca her 'go-to information source' for healthcare news because she valued the commentary provided by community members: "When you want to get the truth in this situation, or what's really going on, I go to \#hcsmca because the people involved are generally there." While most participants described the value of learning from influential people, Chris found the prestigious makeup of the community aligned with his advocacy goals: "My real reason for joining is to try and have influence and to try and make these influential people see things from a more global perspective." These two examples show the direction of information flow in this community - @ carrield99 is receiving information from the community, whereas Chris is promoting views to the community. This twoway information flow provides the opportunity for more generalized reciprocity in exchanges. Conversations are not just one-on-one; rather, the community is seeded with information and opinions, both which add to the social capital of the network.

Getting feedback from community members was also described as an advantage of tapping into the social network of \#hcsmca. Most participants who cited feedback were interested in receiving it to inform their projects and ideas. However, Jessica describes how watching the feedback others receive was also of value to the small community practice she represented: "it sometimes turns into an informal focus group and we get this kind of sample experience from the feedback that's being shared and we can apply that to our own knowledge." This aspect exemplifies again the role of social capital, i.e., in the knowledge held in the network rather than in the 'head' of any particular individual. 
4.1.2 Developing relationships. While developing relationships with other community members was mentioned less frequently than tapping into the social network, it emerged as an important feature across interviews. Participants were asked if they had developed a relationship with any other community members, if that relationship was personal or professional, and if they considered any of these relationships to be close. Half the participants described developing relationships with community members, making both personal and professional connections. Professional relationships were more common. Of the participants who made professional connections, half described them as strong, using terms such as "close colleague" to describe their relationship.

Personal relationships were less common and for many participants the closeness of personal relationships was challenging to define. For example, (a) seastarbatita stated:

"A close friend is a pretty tight thing for me . . I've made friends [through \#hcsmca] that if I'm having a hard time, I'll message them and say 'Hey, things are really rough right now.' I Skype a couple of them to chat and I've met them in person and I've had them . . . to stay in my house. I've made really good connections of people that I care about. Not just people that I know and would say hi to."

The personal and professional connections developed through participation in the community sometimes continued beyond Twitter, with many connecting online through email, Skype, or LinkedIn (but rarely Facebook, a site reserved for offline friends and family) or offline, often through scheduled \#hcsmca meet-ups. @drpauldempsey from (a) quintepediatric described how participating in the Tweet-chats was a satisfactory way to get to know others in the community:

"When I met [community member] for the first time he and I were sort of marveling that it was the first time that we'd met face to face. I said to him that the reason that it seems so strange is because I know you so well. There wasn't anything from that conversation or from meeting him face-to-face that was kind of jarring. I already knew him so well."

The offline meet-ups, including the offshoot offline community EveningRounds, were popular with community members who described them as fun and productive events which helped galvanize their online participation. In keeping with other findings about how closer ties use more media to communicate (media multiplexity), off-Twitter connections were often associated with strong professional and personal relationships. Of the ten participants who described making strong relationships through participation, nine had connected with other participants outside of Twitter and seven had connected in multiple ways.
4.1.3 The community ethos. Analysis showed that community ethos was the third social driver for participation in \#hcsmca. Over half of the participants mentioned some aspect of the community that was important to their participation. These included friendliness, supportiveness, and generosity of the community as well as the well-run nature of \#hcsmca, including leadership by Young. All these factors contribute to expressions of trust in the community.

\#hcsmca was described as welcoming, inclusive, and respectful; as summed up by @NatriceR: “it doesn't matter what walk of life I come from or even if I'm just a patient or a family member or something; it's still a place where anybody can come and listen." Others describe supportiveness, often in relation to their own contributions. For example, Andrew states: "the group is very supportive especially if you have a bright idea." This is also reflected in referring to the generosity of the community and its members. For example, one participant described community members as generous with their knowledge as they were always willing to share their expertise; another that community members were caring and always willing to help others.

The friendliness, support, and generosity of the community are factors that contribute to trust. Getting to know other community members meant that others had become trustworthy sources of information. Trust developed online was found to extend offline. Young shared a story that illustrates this: on a trip to the United Kingdom she visited a friend she had met via Twitter. Upon arrival the friend had to leave to run an errand, leaving Young alone in her home for an hour after meeting in person for approximately 10 minutes. For all intents and purposes Young was a stranger, yet the friend trusted Young in her home; the friend returned home not to find it ransacked, but to a table set with wine and dinner.

The final community-based components cited as important relate to how the community is run. The impact of organization was described by @NatriceR: "I think that it has a very high standard for their discussions and for the way they run it and I really admire that. There's a real consistency and continuity." In a sense, this also extends the idea of trust, as participants trust that their time is well-spent with \#hcsmca.

As Young was the driving force behind the community, it's organization has been inextricably entwined with her leadership; her role in the community was cited by one third of participants as an important element of their participation. For example, Jessica lauded Young for her community management skills, describing the significance of Young's work revitalizing the community through eliciting feedback 
from members and adapting the community: "I have to say that as far as Twitter chats go, hesmca is definitely one of the best ones going."

In addition to organization, Young often acted as a bridge between community members. Her role as a connector was important to Kelly:

"throughout the week she is active in saying 'this person' or that person, or she's connecting with this person, or she's like 'oh, this is a good idea, what do you think, Kelly?' That's more where I see the value"

As someone who spans multiple social networks Young has been an important bridge through which new people, information, and ideas enter the community [5].

Young's role as a leader and connector was advantageous for the entire community, but the group is then highly affected by her departure. During earlier interviews with Young, she expressed a desire to take the focus off her and put it more to the community e.g., in having guests lead the tweet chats. This succeeded in distributing conversations while she was present (see [12]). The legacy may be what allows transformation now, after five years of operation, from \#hcsmca to the EveningRounds community.

\subsection{Relationships between participation and social drivers}

To respond to the second research question, "How do different elements of participation relate to social motivations?" axial analyses were conducted using the social motivators that emerged from the thematic analysis presented above and three facets of participation: length, depth, and frequency. The results are presented as a series of portraits that are grounded in participants' perspectives of their motivations for participating and perceptions of their participation in the community.

4.2.1 Portraits by length. Tapping into the \#hcsmca social network was an important motivation for all Newbies; only one cited relationship development and another community ethos. All Newbies described having the opportunity to be heard, suggesting that new members see \#hcsmca as a forum in which they can share their expertise. Connecting with others who share a common interest and engaging with prestigious healthcare professionals were also highly cited motivators, suggesting that despite participating the community for a short time, Newbies participate in the exchange of social capital: they contribute to the community by adding their voices to the discussion and benefit through interactions with experts.

The social network was also the most commonly cited motivation among Regulars. However, unlike
Newbies, half of the Regulars cited relationship development and community ethos, suggesting that time spent in the community plays a role for these two motivations. Of the network motivators, Regulars most commonly cited connecting with people who share a common interest, getting an overview of the field, and access to diversity. For Regular participants, orientation in the field is an important aspect of participation; as they learn who is who and who knows what, they can hone in on and tap into diverse sources of information and knowledge.

Veterans cited the social network, relationship development, and community ethos with nearly the same frequency. Time appears to play a role in the development of motivations; unsurprisingly, participants who had been members the longest had time to develop relationships; these findings are similar to those of [26], who found that long-time Flickr users had more contacts and belonged to more groups. Veterans' relationships were both personal and professional and the majority were described as strong. There are two possible explanations for the importance of community ethos among Veterans: those who enjoy the community are more likely to continue participating, and/or the impact of the community's structure and ethos becomes more salient over time.

4.2.2 Portraits by depth. For Readers, tapping into the social network was a key motivator. The two most commonly cited aspects of the network were getting an overview of the field and connecting with people with similar interests. Both of these are motivations that can be achieved through passive participation and are reflective of legitimate peripheral participation [23], a process which provides opportunities to learn the culture of the community and practice, and to make that practice their own.

Accessing the network was also the most commonly cited motivator of Contributors. While Contributors were not motivated by relationship development, a third cited the community ethos as important to their membership. Similar to findings by [27], despite low levels of participation Contributors felt as though they were part of the community. Interestingly, of the network motivators, being heard was the most commonly cited by Contributors. According to [31], community members engage in low levels of participation as they take time to "get to know" the community that provides a latent tie structure [13]. For these contributors, the opportunity to be heard is important - as they familiarize themselves with the community they have the potential to move from contribution to collaboration and activate latent tie relationships. 
Collaborators were almost equally motivated by accessing the social network, relationship development, and community ethos. Relationships developed by Collaborators were both personal and professional, strong and weak. Of the network motivators, connecting with people who have shared interests and being heard were most frequently cited, followed by diversity. While shared interests and being heard were motivations Collaborates shared with Contributors, diversity as a motivator was much more commonly cited by Collaborators. This suggests that there is an association between depth of participation and diversity - participants who interact with others in meaningful ways are able to gain more insight into the expertise and roles of other community members.

All of the Leaders were motivated by accessing the social network, relationship development and community ethos. Relationships were primarily described as professional; however, all were considered close. Of the network motivators, all were motivated by connecting with people with shared interests and diversity; being heard, getting an overview of the field, and feedback were cited by two thirds. As with Collaborators, Leaders benefited from both bridging and bonding social capital; depth of contribution was associated with higher degrees of bonding capital made through the development of strong relationships.

4.2.3 Portraits by frequency. Community members who tuned into the chats Rarely were motivated by access to the social network, with one participant motivated by relationship development, and one by community ethos. Of the network motivators, the most commonly cited was connecting with people who share a common interest, followed by being heard and getting an overview of the field. This suggests that as with Contributors, being heard is reflective of intention rather than behaviour - those who participate rarely know that when they do participate, their voices will be heard.

Community members who participated Sporadically were most motivated by accessing the network; however, approximately two thirds cited community ethos while half cited relationship development. Sporadic participants described developing strong and weak personal and professional relationships. Of the network motivators, overview of the field, connecting to people with a shared interest, and diversity were all common motivators. However, the most commonly cited motivator was being heard. For those who participate sporadically, participating to be heard is likely related to self-efficacy [1]. Many participants stated that they were more likely to actively contribute when topics appealed to them, when they felt they had something of value to contribute, or could confidently share their expertise. The relationship between self-efficacy and participation has been found in other knowledge sharing communities [e.g., 18, 31].

Community members who participated Habitually were all motivated by access to the network and just over two thirds were motivated by relationship development and community ethos. As with community members who participate sporadically, these relationships were both personal and professional; however, all habitual participants described these relationships as strong. This suggests that ritual participation is associated with building strong bonds. Of the network motivators, connecting to people with a shared interest was most common, followed by being heard, diversity and feedback. Habitual participation provides these community members with regular access to information as well as a space in which they can develop their ideas with input from others.

\section{Discussion}

Patterns of participation across the three facets, length, depth, and frequency, roughly correspond to light and heavyweight models of participation [14]: Newbies, Readers, Contributors, and members who participate rarely are associated with lightweight participation; while Regulars, Veterans, Contributors, Leaders, and members who participate habitually are associated with heavyweight participation. Lightweight participation in \#hcsmca was associated with weak tie relationships and the predominantly bridging social capital it affords: participants accessed new information by connecting with people who have a shared interest, were able to use these connections to see an overview of the field, and interact with prestigious people in healthcare. Likewise, in addition to bridging social capital, heavyweight participation was associated with strong tie relationships and the predominantly bonding social capital it affords: through interactions in the well-organized and supportive community, participants developed trusting personal and professional relationships that extended beyond Twitter. While [17] found higher levels of bridging social capital among Twitter users, findings from this study suggest that heavyweight participation in Twitter-based communities can facilitate the development of rich bonding capital.

Early characterizations of lightweight participants, described them as "free loaders" who used a common good (the community) without giving back [20]. However, findings here support work by [27, 28], which suggests that lightweight participation provides 
an essential aspect of an online community's vitality: through their desire to be heard, Newbies brought novel information to the network [11] and had the potential to act as bridges between \#hcsmca and other social networks [5]. These benefits are illustrated by Rajiv (@DrRKSingal): “even though I wander in and out a little bit there's new people that seem to be on every time so there's an opportunity to continue to learn and figure out different perspectives." Readers, and Contributors engaged in legitimate peripheral participation [23] as they learned about the topic, the community, and its norms. Several lightweight participants described contributing to the discussion when the topic was in their area of expertise, suggesting that even through minimal participation, lightweight participants in \#hcsmca contributed to the generalized reciprocity associated with the exchange of bridging and bonding capital. A community in which membership ebbs and flows as new members enter and as latent ties are activated will support the introduction of fresh perspectives and new ideas.

Generally, patterns between participation and motivation in \#hcsmca fit the light/heavyweight model; however, two deviations from the norm were observed: sporadic participation, and diversity as a motivation. In their study of a citizen science project, [9] found that sporadic contributors had motivations and participation patterns associated with lightweight participation. However, sporadic participants in \#hcsmca described much the same motivators as those who engaged in heavyweight participation. Interviews with Sporadic participants suggests that their participation is affected by two factors: community structure and time available. As the community discussed a different topic every week, not all topics were equally appealing to all members; many participants explained that they would not follow or participate in chats if the topic did not appeal to them. Time was also often associated with sporadic participation; community members did not always have time to participate or had scheduling conflicts. That these members were able build bonding capital shows that in \#hcsmca, habitual participation was not a requisite for the development of bonding capital.

The second deviation from the light and heavyweight model is diversity as a motivation. Typically, motivations derived from strong tie connections, such as relationship development and community ethos, were cited by heavyweight participants; and motivations derived from weak tie connections, such as interacting with others who share a common interest and getting an overview of the field, were were cited by light and heavyweight participants. However, while diversity is commonly associated with weak tie relations [11] it was only cited as a motivation by heavyweight participants. These findings suggest that while social network diversity is enacted through weak tie relations, it takes sustained and/or substantial participation in a network to see its benefits.

Overall, patterns of participation generally align with the light and heavyweight model of participation. These patterns support the framework described by [14] and are aligned with findings by [4] and [9]. Thus, in \#hcsmca, the light and heavyweight model can be used to explain motivational aspects of the community. However, individual participants will often cross boundaries between weights - for example, one participant was a Newbie, Collaborator, and participated habitually; therefore, examining motivations by facets of participation provides community leaders and designers with a nuanced foundation for building successful communities through meeting the motivations of community members who participate in different ways and to varying degrees.

\section{Conclusion}

The current work set out to explore the social motivations for participating in the Twitter-based online community of practice, \#hcsmca, and the relationship of social motivations to various facets of participation. Analysis of interviews with 24 community members found that social motivators played a significant role in participation in \#hcsmca and provided them with opportunities to build bridging and bonding social capital. This research also explored the relationship between motivations and three facets of participation: length, depth, and frequency. These facets were used to develop motivational portraits of participation. Typically patterns of participation corresponded to light and heavyweight models of participation and provide further empirical support for the light/heavyweight model [14]; however, contrary to findings in prior research [9], sporadic participants shared similar social motivations as heavyweight participants, suggesting that there may also be a relationship between the type of online initiative and motivation [14]. This link will be explored in future research. This study is limited by its purposive sampling; it is likely that interviewees do not represent a cross section of the community. However, purposive sampling provided insight into the perspectives of participants at various types and levels of participation.

In addition to providing a nuanced account of participation that can be used by community designers to meet the needs of their members, these results contribute to theoretical perspectives on community development by providing additional empirical support for the light and heavyweight model of participation, 
and fills a gap in the literature by addressing diverse elements of participation.

\section{References}

[1] A. Bandura, Self-efficacy: The exercise of control, New York: W.H. Freeman, 1997.

[2] Y. Benkler, The penguin and the leviathan: The triumph of cooperation over self-interest, New York: Crown Business, 2011.

[3] D. Brabham, "Motivations for participation in a crowdsourcing application to improve publish engagement in transit planning," J. of Appl. Commun. Research, vol. 40, pp. 307-328, Jun 2012.

[4] N. Budhathoki and C. Haythornthwaite, "Motivation for Open Collaboration: Crowd and Community Models and the Case of OpenStreetMap," Amer. Behavioral Scientist, vol. 57, pp. 548-575, Dec 2012.

[5] R.S. Burt, "Structural holes and good ideas," Amer. J. of Sociology, vol. 110, pp. 349-399, Sept 2004.

[6] Canadian Instit. of Health Research, Natural Sci. and Eng. Research Council of Canada, and Social Sci. and Humanities Research Council of Canada, "Tri-Council Policy Statement: Ethical Conduct for Research Involving Humans," 2014

[7] J. Corbin and A. Strauss, Basics of qualitative research: Techniques and procedures for developing grounded theory, 3rd Edition, Thousand Oaks: Sage, 2008.

[8] N. B. Ellison et al., "The benefits of Facebook 'friends:' Social capital and college students' use of online social network sites," J. of Comput. Mediated Commun., vol. 12, pp. 1143-1168, Aug 2007.

[9] A. Eveleigh et al., "Designing for dabblers and deterring drop-outs in citizen science," in Proc. of CHI, 2014, pp. 2985-2994.

[10] S. Gilbert, "Learning in a Twitter-based community of practice: an exploration of knowledge exchange as a motivation for participation in \#hcsmca," Inform., Commun., and Soc., vol. 19, pp. 1214-1232, May 2016.

[11] M. S. Granovetter, "The strength of weak ties," Amer. J. of Sociology, vol. 78, pp. 1360-1380, May 1973.

[12] A. Gruzd and C. Haythornthwaite, "Enabling Community through social media", J. of Medical Internet Research, vol 15, Oct 2013.

[13] C. Haythornthwaite, "Social networks and Internet connectivity effects", Inform., Commun., and Soc., vol. 8, pp. 125-147, Jun 2005.

[14] C. Haythornthwaite, "Crowds and communities: Light and Heavyweight models of peer production," in Proc. of HICSS, 2009, pp. 1-10.

[15] C. Haythornthwaite et al. "A social network analytic perspective on e-learning," In, Handbook of E-Learning Research. C. Haythornthwaite et al., Eds. London: Sage. 2016, pp. 251-269.

[16] I. Himelboim et al., "Birds of a feather tweet together: Integrating network and content analyses to examine crossideology exposure on Twitter," J. of Comput. Mediated Commun, vol. 18, pp. 154-174, 2013.

[17] M. Hofer and V. Aubert, "Perceived bridging and bonding capital on Twitter: Differentiating between follower and followers and followees", Comput. in Human Behavior, vol. 29, pp. 2134-2142, Nov 2013.

[18] S. Hung et al., "Knowledge sharing intention in professional virtual communities: A comparison between posters and lurkers," J. of the Assoc. for Inform. Sci. \& Technology, vol. 66, pp. 2494-2510, Dec 2015.

[19] N. Kauffman et al., "More than fun and money. Worker motivation in crowdsourcing - a study on Mechanical Turk," in Proc. of the Amer. Conference on Inform. Syst, 2011, pp. $1-11$.

[20] P. Kollock and M. Smith, "Managing the virtual commons: Cooperation and conflict in computer communities" in Computer-mediated communication: Linguistic, social and cross-cultural perspectives. S. Herring Ed. Amsterdam: John Benjamin, 1996, pp. 109-128.

[21] R. Kraut and P. Resnick, P. "Encouraging contribution to online communities" in Building successful online communities: Evidence-based social design. In R. Kraut et a., Eds. Cambridge: MIT Press, 2011, pp. 21-76.

[22] C. Lampe, et al. "Motivations to participate in online communities" in Proc. CHI, 2010, pp. 1927-1936

[23] J. Lave and E. Wenger, "Legitimate peripheral participation in communities of practice," in Supporting lifelong learning: Vol. 1 perspectives on learning. $\mathrm{R}$. Harrison et al., Eds. London: Routlege, 2002, pp. 111-126.

[24] S. Malinen, "Understanding user participation in online communities: A systematic review of empirical studies," Comput. in Human Behavior, vol. 46, pp. 228-238, Feb 2015. [25] O. Nov. "What motivates Wikipedians?" Comm. of the $A C M$, vol. 50, pp. 60-64, Nov. 2007

[26] O. Nov, et al. "Analysis of participation in an online phot-sharing community: A multidimensional perspective. $J$. of the Assoc. for Inform. Sci. \& Technology, vol. 61, pp. 555566, Oct. 2010.

[27] J. Preece et al., "The top five reasons for lurking: improving community experiences for everyone," Comput. In Human Behavior, vol. 20, pp. 201-223, Mar 2004.

[28] J. Preece and B. Shneiderman, "The Reader to Leader Framework: Motivating Technology Mediated Social Participation", Trans. on Human Comput. Interaction, vol. 1, pp. 13-32, Mar 2009.

[29] R. D. Putnam, Bowling Alone: The Collapse and Revival of American Community. New York: Simon \& Schuster, 2000.

[30] R. M. Ryan and E. L. Deci, "Intrinsic and extrinsic motivations: Classic definitions and new directions," Contemporary Educ. Psychology, vol. 25, pp. 54-67, 2000.

[31] V. Soroka and S. Rafaeli, "Invisible participants: How cultural capital relates to lurking behavior," in Proceedings of $W W W, 2006$, pp. 163-172.

[32] E. Wenger. Introduction to communities of practice. [web page] Available: http://wengertrayner.com/introduction-to-communities-of-practice/

[33] C. Young, C. (2016, Feb 28) \#hcsmca: 5-Year retrospective and looking ahead, [blog post]. Available: https://www.cyhealthcommunications.wordpress.com/2016/0 2/28/hcsmca-5-year-retrospective-and-looking-ahead

[34] H. Zhu et al. "Effects of peer feedback on contribution: a field experiment in Wikipedia" in Proc. of CHI, 2013, pp. 2253-2262. 